\title{
Understanding triangulation in research
}

\section{Roberta Heale, ${ }^{1}$ Dorothy Forbes ${ }^{2}$}

$10.1136 /$ eb-2013-101494

${ }^{1}$ School of Nursing, Laurentian University, Sudbury, Ontario, Canada

${ }^{2}$ Faculty of Nursing, University of Alberta, Edmonton, Alberta,

Canada

Correspondence to: Roberta Heale

School of Nursing, Laurentian University, Sudbury, ON,

Canada P3E2C6;

rheale@laurentian.ca
The term 'triangulation' originates in the field of navigation where a location is determined by using the angles from two known points. ${ }^{1}$ Triangulation in research is the use of more than one approach to researching a question. The objective is to increase confidence in the findings through the confirmation of a proposition using two or more independent measures. ${ }^{2}$ The combination of findings from two or more rigorous approaches provides a more comprehensive picture of the results than either approach could do alone. ${ }^{3}$

Triangulation is typically associated with research methods and designs. However, there are several other variations on the term. Triangulation may be the use of multiple theories, data sources, methods or investigators within the study of a single phenomenon. ${ }^{2}{ }^{4}$ The technique was originally introduced into qualitative research in the 1950s as a means to avoid potential biases arising from the use of a single methodology. ${ }^{4}$ This technique is used to confirm suggested findings, but it can also be used to determine the completeness of data.

Methodological triangulation is the most common type of triangulation. ${ }^{2}$ Studies that use triangulation may include two or more sets of data collection using the same methodology, such as from qualitative data sources. Alternatively, the study may use two different data collection methods as with qualitative and quantitative. "This can allow the limitations from each method to be transcended by comparing findings from different perspectives...."

Triangulation is often used to describe research where two or more methods are used, known as mixed methods. Combining both quantitative and qualitative methods to answer a specific research question may result in one of the following three outcomes: (1) the results may converge and lead to the same conclusions; (2) the results may relate to different objects or phenomena but may be complementary to each other and used to supplement the individual results and (3) the results may be divergent or contradictory. Converging results aim to increase the validity through verification; complementary results highlight different aspects of the phenomenon or illustrate different phenomenon and divergent findings can lead to new and better explanations for the phenomenon under investigation. ${ }^{3}$

Examples of triangulation, or mixed methods, are as varied as there are research studies. Nurses' attitudes about teamwork may be collected through a survey and focus group discussion. A study to explore the reduction of blood pressure through a nutritional education programme may include a review of participant adherence to the diet changes through daily logs along with a series of BP readings. In every case, the researchers link and compare different methods related to a single research question.

Although regarded as a means to add richness and depth to a research inquiry, there are several criticisms of the use of triangulation in research. Triangulation assumes that the data from two distinct research methods are comparable and may or may not be of equal weight in the research inquiry. In addition, when two or more data sets have convergent findings, there must be caution in interpretation since it may simply mean that each of the data sets is flawed. Others ${ }^{3}$ question whether the term triangulation has any meaning when it is so broadly defined, mixed methods is preferred. In spite of these criticisms, triangulation is generally considered to promote a more comprehensive understanding of the phenomenon under study and to enhance the rigour of a research study.

Competing interests None.

\section{References}

1. The Institute of Navigation. (n.d.). Getting to the point. http:// www.ion.org/satdiv/education/lesson6.pdf

2. Bryman A. (n.d.). Triangulation. http://www.referenceworld. com/sage/socialscience/triangulation.pdf

3. Tashakkori A, Teddle C. Handbook of mixed methods in social and behavioral research. Thousand Oaks, CA: Sage, 2003.

4. Williamson GR. Illustrating triangulation in mixed-methods nursing research. Nurse Res 2005;12:7-18. 